\section{JTI}

JOURNAL OF

TRAUMA AND INJURY

\title{
Case Series of Zone III Resuscitative Endovascular Balloon Occlusion of the Aorta in Traumatic Shock Patients
}

\author{
Byungchul Yu, M.D. ${ }^{1,2}$, Gil Jae Lee, M.D., Ph.D. ${ }^{1,2}$, Kang Kook Choi, M.D. ${ }^{1,2}$, \\ Min A Lee, M.D. ${ }^{1,2}$, Jihun Gwak, M.D. ${ }^{1}$, Youngeun Park, M.D. ${ }^{1}$, \\ Jung Nam Lee, M.D., Ph.D., \\ ${ }^{1}$ Department of Trauma Surgery, Gachon University Gil Medical Center, Incheon, Korea \\ ${ }^{2}$ Department of Traumatology, Gachon University College of Medicine, Incheon, Korea
}

Received: July 27, 2020

Revised: September 9, 2020

Accepted: September 15, 2020

\section{Correspondence to}

Jung Nam Lee, M.D., Ph.D.

Department of Traumatology, Gachon University Gil Medical Center, 783

Namdong-daero, Namdong-gu, Incheon

21565, Korea

Tel: $+82-32-460-3010$

Fax: +82-32-460-3247

E-mail:jnlee@gilhospital.com
Purpose: There is increasing evidence in the literature regarding resuscitative endovascular balloon occlusion of the aorta (REBOA) globally, but few cases have been reported in Korea. We aimed to describe our experience of successful Zone III REBOA and to discuss its algorithm, techniques, and related complications.

Methods: We reviewed consecutive cases who survived from hypovolemic shock after Zone III REBOA placement for 4 years. We reviewed patients' baseline characteristics, physiological status, procedural data, and outcomes.

Results: REBOA was performed in 44 patients during the study period, including 10 patients (22.7\%) who underwent Zone III REBOA, of whom seven (70\%) survived. Only one patient was injured by a penetrating mechanism and survived after cardiopulmonary resuscitation. All patients underwent interventions to stop bleeding immediately after REBOA placement.

Conclusions: This case series suggests that Zone III REBOA is a safe and feasible procedure that could be applied to traumatic shock patients with normal FAST findings who receive a chest X-ray examination at the initial resuscitation.

Keywords: Trauma; Trauma centers; Shock, hemorrhagic; Intra-aortic balloon occlusion

\section{INTRODUCTION}

The first case of the intra-aortic balloon catheter occlusion in a trauma case was described in 1954; however, this technique was not widely utilized until recent years [1]. (http://creativecommons.org/licenses/by-nc/4.0/) which permits unrestricted noncommercial use, distribution, and reproduction in any medium, provided the original work is properly cited. 
With advances in endovascular techniques, resuscitative endovascular balloon occlusion of the aorta (REBOA) is becoming a procedure of choice for many trauma surgeons. REBOA can be used for non-compressible torso hemorrhage in patients with hypovolemic shock. It is known to preserve cerebral and cardiac perfusion, in addition to decreasing distal hemorrhage. Aortic clamping with emergency thoracotomy has been used for the same purpose, but it carries the risk of tissue damage and a high morbidity rate $[2,3]$.

Generally, the accepted indication of REBOA is life-threatening hemorrhage below the diaphragm in injured patients who are in an unresponsive or transiently responsive state of shock. Furthermore, REBOA is indicated for patients with cardiac arrest due to suspected torso hemorrhage, but there is lack of evidence regarding the recommended duration of arrest and the benefits of REBOA compared with thoracotomy [4]. An aortic balloon can be inflated at the distal thoracic aorta (Zone I) or the distal abdominal aorta (Zone III) depending on the injury site. Zone III REBOA is indicated for patients with severe pelvic, junctional, or proximal lower extremity hemorrhage. In addition, Zone III REBOA can be inflated for a longer time with fewer complications than Zone I REBOA.

In Korea, the first case of REBOA for a trauma victim was reported in 2015, and since then, several trauma centers have implemented this procedure [5]. In 2015, Incheon regional trauma center implemented instruments and a protocol for REBOA, leading to its practice by surgeons after simulation-based training. Our first case was that of a patient with profound hypovolemic shock due to hemoperitoneum and pelvic fracture in December 2015. We then performed REBOA in 24 cases during the next 4 years, as described in a previously published article [6]. Recently, a pioneering group of surgeons at Dankook University in Korea have developed an endovascular training for REBOA as a basic course, with guidance regarding its implementation at domestic trauma centers [7].

In this report, we describe our experience of successful deployment of Zone III REBOA and its possible indications. In addition, we discuss the outcomes of REBOA, its technical aspects, appropriate algorithms, and complications.

\section{METHODS}

\section{Patients}

We reviewed a case series of consecutive trauma patients who survived hypovolemic shock after Zone III REBOA deployment and proper interventions to stop bleeding between January 2016 and December 2019. Patients whose REBOA position was changed (Zone III to I, Zone I to III) were excluded from this study. We reviewed patients' baseline characteristics and physiological status, including the initial and the lowest systolic blood pressure (SBP), SBP after REBOA inflation, heart rate, initial fluids, blood transfusion, time course, and complications related to the procedure. Laboratory data such as hemoglobin, $\mathrm{pH}$, lactate, and base deficit were collected, and trauma scores were calculated with known formulas. Gachon University Institutional Review Board (approval number: 2018-037) waived the requirement to obtain informed patient consent because we used only existing materials and documents. Data were collected and processed anonymously.

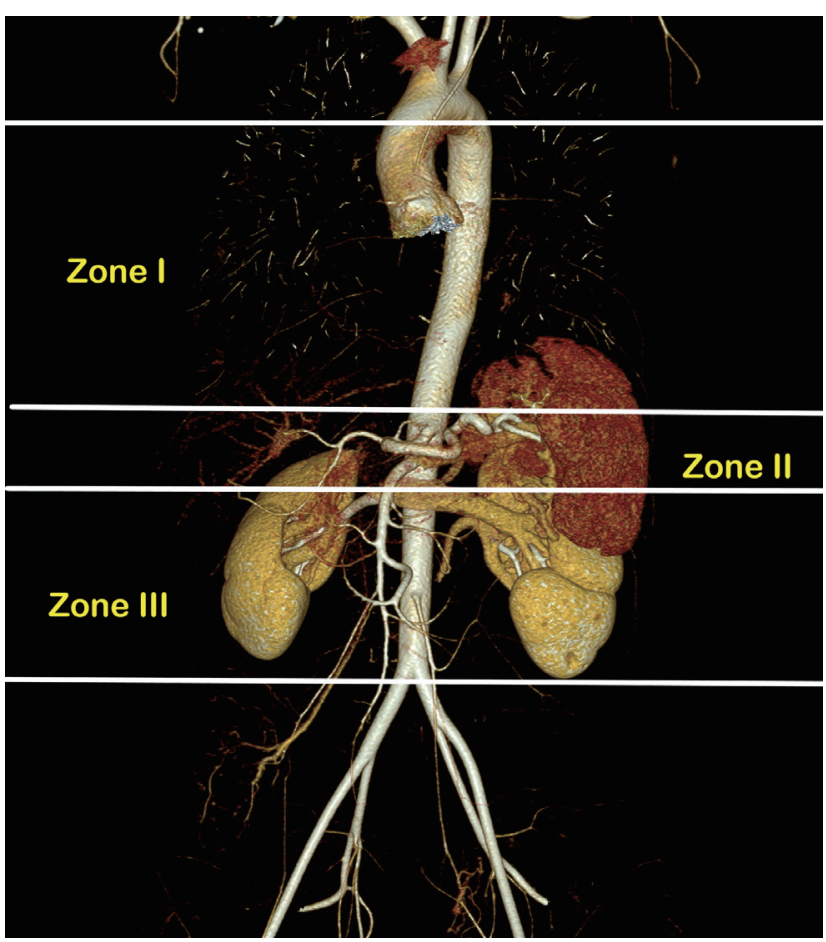

Fig. 1. Division of aortic Zones regarding resuscitative endovascular balloon occlusion of the aorta. 


\section{REBOA candidates and details of the procedure}

To identify traumatic shock patients who were candidates for REBOA, shock was defined as SBP $<90 \mathrm{mmHg}$, a shock index $>1.3$, or base excess $>5$. Patients in shock who did not respond to the initial resuscitation with a liter of crystalloid and two units of red blood cell (RBC) transfusion, or with impending cardiac arrest, were considered candidates for REBOA. Contraindications for the procedure included cardiac arrest for more than $10 \mathrm{~min}$ utes, mediastinal widening, and cardiac tamponade. The institutional indications for REBOA were initially agreed upon, but not formally defined, and the trauma surgeon in charge made the decisions.

The aortic Zones were defined as Zones I, II, and III in craniocaudal succession (Fig. 1). Zone I is at the descending thoracic aorta between the origin of the left subclavian and celiac arteries. Zone II is at the para-visceral aorta from celiac arteries to the lowest renal artery and Zone III at the abdominal aorta between the lowest renal artery and the aortic bifurcation. Zone I was preferred in most cases of profound shock and impending cardiovascular collapse. Zone III was recommended in patients with a pelvic injury or junctional femoral injury without thoraco-abdominal injury. We avoided Zone II in all circumstances due to severe splanchnic ischemia after balloon inflation.

Trained trauma surgeons inserted all of the aortic occlusion balloons without radiological assistance. A 7-Fr or 12 -Fr sheath was inserted into the common femoral artery, and its size was selected depending on the balloon catheters available. During the study period, two kinds of balloon catheters were available. The CODA balloon catheter (Cook Incorporated, Bloomington, IN, USA) is an over-the-wire 12-Fr device with a balloon volume of $60 \mathrm{~mL}$, while the RESCUE balloon catheter (Tokai Medical Products, Aichi, Japan) is a smaller device (7 Fr) with a compliant balloon up to $40 \mathrm{~mL}$. There was a written procedure protocol, and some trauma surgeons in Incheon regional trauma center participated in REBOA and endovascular training courses in Korea; however, initially, only a few trained surgeons could perform the procedure. Incheon regional trauma center started a training course for vascular and endovascular skills for trauma surgeons in 2017, and since then, all trauma surgeons have been

Table 1. Clinical characteristics

\begin{tabular}{|c|c|c|c|c|c|c|c|}
\hline Patient & 1 & 2 & 3 & 4 & 5 & 6 & 7 \\
\hline Age & 20 & 44 & 73 & 20 & 33 & 45 & 62 \\
\hline Sex & Male & Male & Female & Female & Male & Female & Male \\
\hline Mechanism of injury & Fall & Fall & MVC & Fall & Stab & Fall & MVC \\
\hline Injury severity score (ISS) & 35 & 27 & 26 & 22 & 16 & 38 & 16 \\
\hline Cardiac arrest before REBOA & No & No & No & No & Yes & No & No \\
\hline FAST & $(-)$ & $(-)$ & $(-)$ & $(-)$ & $(-)$ & $(-)$ & $(-)$ \\
\hline Initial SBP (mmHg) & 60 & 103 & 60 & 59 & 0 & 143 & 80 \\
\hline Lowest SBP (mmHg) & 60 & 48 & 52 & 43 & 0 & 50 & 60 \\
\hline Increased SBP after REBOA (mmHg) & 68 & 72 & 36 & 29 & 40 & 49 & 36 \\
\hline Initial pH & 7.39 & 7.2 & 7.28 & 7.0 & 7.05 & 7.32 & 7.2 \\
\hline Initial base deficit & 4.8 & 11.9 & 11.9 & 15 & 20.3 & 9.4 & 18 \\
\hline RBC transfusion within 4 hours (unit) & 10 & 19 & 10 & 8 & 20 & 5 & 12 \\
\hline FFP transfusion within 4 hours (unit) & 5 & 3 & 8 & 4 & 8 & 0 & 8 \\
\hline RBC transfusion within 24 hours (unit) & 16 & 26 & 21 & 8 & 20 & 7 & 12 \\
\hline FFP transfusion within 24 hours (unit) & 16 & 17 & 17 & 8 & 14 & 3 & 12 \\
\hline
\end{tabular}

REBOA: resuscitative endovascular balloon occlusion of the aorta, FAST: focused abdominal assessment for trauma, MVC: motor vehicle collision, SBP: systolic blood pressure, RBC: red blood cell, FFP: fresh frozen plasma. 
capable of performing REBOA.

\section{RESULTS}

During the study period, REBOA was performed in 44 patients, including cardiac arrest patients. Zone III REBOA was performed in 10 patients (22.7\%), of whom seven $(70 \%)$ survived profound hypovolemic shock. The clinical characteristics of the patients are displayed in Table 1. Only one patient was injured by a penetrating mechanism and this patient experienced pulseless electrical activity in the trauma bay. The mean age and injury severity score (ISS) were 45.4 years and 25.7, respectively. Focused abdominal assessment for trauma (FAST) findings were negative in all patients. The SBP increased after REBOA insertion from $29 \mathrm{mmHg}$ to $72 \mathrm{mmHg}$. All pa- tients received more than five units of $\mathrm{RBC}$ transfusion in the first 4 hours after admission.

REBOA-related characteristics and outcomes are shown in Table 2. We used a 12-Fr balloon catheter in one patient and for each of the others, we used a 7-Fr balloon catheter. The mean time of REBOA deployment from emergency department arrival was 30.5 minutes. The major source of bleeding was pelvic bone fracture in five patients and junctional vascular injury in two patients. In one patient, we performed preperitoneal pelvic packing (PPP) to control bleeding and angioembolization was performed in two patients. Both PPP and angioembolization were performed in two patients, and direct surgical vascular repair in the operation room was performed in two patients. There was one procedure-related complication, which was femoral artery thrombosis after sheath removal. The patient underwent immediate surgical

Table 2. Procedure-related data and outcomes

\begin{tabular}{|c|c|c|c|c|c|c|c|}
\hline Patient & 1 & 2 & 3 & 4 & 5 & 6 & 7 \\
\hline Balloon catheter (Fr) & 12 & 7 & 7 & 7 & 7 & 7 & 7 \\
\hline Source of bleeding & Pelvis & Pelvis & Pelvis & Pelvis & Femoral vessels & Pelvis & Femoral vessels \\
\hline Interventions after REBOA & $\begin{array}{l}\text { PPP } \\
\text { AE }\end{array}$ & $\begin{array}{l}\text { PPP } \\
\text { AE }\end{array}$ & $\mathrm{AE}$ & PPP & Surgery & $\mathrm{AE}$ & Surgery \\
\hline Complications & No & $\begin{array}{l}\text { AKI } \\
\text { SSI }\end{array}$ & No & No & No & No & No \\
\hline Procedure-related complications & CFA thrombosis & No & No & No & No & No & No \\
\hline Outcomes & $\begin{array}{c}\text { Survive } \\
\text { Amputation }\end{array}$ & Survive & Survive & Organ donation & Survive & Survive & Survive \\
\hline
\end{tabular}

REBOA: resuscitative endovascular balloon occlusion of the aorta, PPP: preperitoneal pelvic packing, AE: angioembolization, AKI: acute kidney injury, SSI: surgical site infection, CFA: common femoral artery.
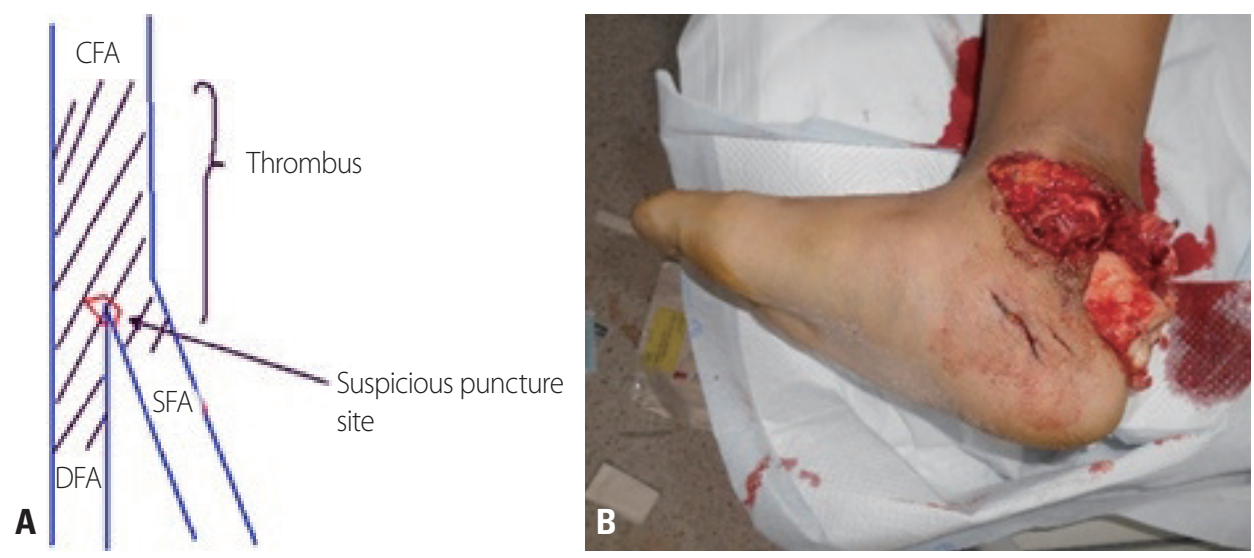

Fig. 2. (A) Operative findings of right femoral artery thrombosis in patient \#1. (B) Open calcaneal fracture of the right ankle in patient \#1. CFA: common femoral artery, DFA: deep femoral artery, SFA: superficial femoral artery. 
thrombectomy, but the patient was discharged later after below-knee amputation (Fig. 2). A large profile balloon and sheath were used in this patient. All patients survived and were discharged after proper management except one who died after organ donation due to severe traumatic brain injury.

\section{DISCUSSION}

In this report, we described the characteristics and outcomes of patients who underwent a 'pure' Zone III REBOA procedure. Our findings suggest that Zone III REBOA could be applied to traumatic shock patients with normal FAST findings who receive a chest X-ray at the initial resuscitation phase. This could be safer and more feasible than Zone I REBOA.

\section{REBOA algorithm and selection of the target zone}

Most algorithms recommend femoral artery access or REBOA for hypotensive (SBP $<90 \mathrm{mmHg}$ ) patients with partial or no response to initial fluid or blood product resuscitation $[8,9]$. However, definitions of hemodynamic instability and available resources vary considerably across trauma centers. Therefore, algorithms should be tailored to the available resources and expertise at individual centers [10]. For example, expert vascular surgeons or interventional radiologists can perform REBOA in a few minutes, but a surgeon with less experience may experience delays in definitive procedures such as laparotomy, pelvic packing, and embolization. In that case, resuscitative thoracotomy-aortic cross clamping (RT-ACC) or rapid transportation to the operation room could be a life-saving procedure. The more the hemodynamic status deteriorates, the more difficult common femoral artery access becomes. Therefore, achieving early access with a smaller arterial line and upsizing to larger sheath for REBOA placement can be beneficial [11]. For this reason, the recommended algorithm at Incheon regional trauma center involved early access for partial responders with a $4-\mathrm{Fr}$ sheath and upsizing (if needed during arterial monitoring), and direct 8-Fr sheath placement for non-responders. In the future, a low-profile balloon, training course development, and prehospital usage of REBOA could enhance the indications for this procedure and change the algorithm [12,13].

The inflation of the balloon in the three zones has been illustrated clearly in many studies, and most authors recommend two possible zones in the aorta for balloon inflation $[8,14]$. Zone I placement is indicated for patients with FAST or CT-positive abdominal injuries, while Zone III is recommended for patients with hemorrhage due to pelvic injuries or junctional vascular injuries. Generally, algorithms recommend FAST and pelvic X-rays to select the proper occlusion zone in traumatic shock patients $[4,15]$. Zone II occlusion was avoided because the visceral arteries supplying the gastrointestinal tract, liver, and kidney originate there. In theory, Zone I occlusion still permits collateral or retrograde perfusion of the abdominal viscera. This makes Zone I occlusion the preferred zone in the initial phase [16]. Some authors argue that Zone III REBOA provides only minimal hemodynamic support when compared to Zone I REBOA. For this reason, Zone I REBOA should be performed initially in traumatic shock patients to prevent circulation collapse [17]. However, Zone I occlusion can cause widespread organ ischemia, placing a limitation on the occlusion time. In contrast, Zone III balloon placement can effectively reduce bleeding from pelvic fractures or junctional vessels, while minimizing the ischemic burden. In addition, it is relatively easy to perform technically. Although this study presents a small case series, Zone III REBOA was feasible and safe for isolated pelvic and junctional hemorrhages.

\section{Outcomes after REBOA}

Aortic occlusion (either REBOA or RT-ACC) provides several beneficial effects in patients experiencing profound shock. These procedures elevate the central arterial pressure and improve the cerebral and myocardial perfusion rates. In addition, active bleeding below the occlusion point decreases. Several studies have compared RT-ACC with REBOA and reported that REBOA showed similar beneficial effects and improved survival with fewer complications $[18,19]$. Therefore, in patients without cardiac arrest, REBOA is more appropriate as the intervention of choice. Although increasingly many studies have investigated REBOA utilization, solid evidence of its survival benefits is lacking. A recent systemic review showed a 
significant rise in blood pressure and a survival rate of approximately $40 \%$ with patients in shock, although most data were obtained from case reports or observational studies [20]. In the future, the results of combined prospective data collection by the American Association for the Surgery of Trauma registration (AORTA), the European registry (ABOT), the Japanese registry (DIRECT-IA$\mathrm{BO})$ and the UK-REBOA randomized controlled trial will report data on the indications, outcomes, and complications of REBOA [20]. Therefore, the Korean Society of Traumatology should establish a proper REBOA registry, which will eventually be included in the global registry in the future as well.

\section{Procedure-related complications}

In our series, one patient experienced a procedure-related complication (common femoral artery thrombosis). We suspected that this complication was a consequence of improper puncture of the femoral artery and use of a large bore (1-Fr) sheath. The patient underwent below-knee amputation during admission, but we could not confirm whether this procedure was directly related to the thrombotic complication because there was a severe open fracture of the ankle. The most common causes of complications were vascular injuries during arterial access and sheath positioning, which occurred in $30 \%$ of cases with larger devices $[9,21,22]$. Therefore, we recommend using an ultrasonography (USG)-guided puncture technique for vascular access in REBOA. In addition, previous studies have revealed that a low-profile catheter, such as a 7-Fr sheath, led to fewer access-related complications despite the longer duration of sheath placement $[12,23]$. In summary, an appropriate arterial access technique such as USG-guided puncture and low-profile balloon utilization could minimize catheter-related complications. Other complications related to perfusion after REBOA include acute kidney injury (AKI), leg ischemia with embolism, multiple organ failure, and reperfusion syndrome [9]. Some studies have suggested that Zone I occlusion for over 60 minutes could result in significant organ damage, such as acute tubular necrosis and spinal cord injury $[24,25]$. In contrast, several studies have suggested that partial, intermittent Zone III REBOA could minimize such complications [24,26-28]. Therefore, we recommend intermittent or partial REBOA to avoid complete occlusion of the aorta for over 60 minutes and the appropriate usage of Zone III REBOA. In this series, one patient suffered from AKI requiring dialysis, but the patient fully recovered without sequelae during admission.

\section{Limitations}

First, we could not compare cases of traumatic hypovolemic shock with or without REBOA to evaluate the outcomes. Second, because we collected data retrospectively, it was difficult to document all data related to the procedures. In particular, the durations of inflation and deflation were not always recorded accurately. We are now trying to establish a prospectively collected registry for REBOA. Third, before training courses were offered for trauma surgeons, only some surgeons were able to perform REBOA, suggesting that more patients were candidates for REBOA. Therefore, there was an obvious selection bias.

\section{CONCLUSION}

REBOA is a beneficial adjunct for hypovolemic traumatic shock patients with torso hemorrhage. Of particular note, Zone III REBOA is more feasible, safer, and easier to perform than Zone I REBOA in selected cases. Algorithms that properly describe indications and zone selection should be confirmed by well-designed research in the future.

\section{REFERENCES}

1. Dumas RP, Holena DN, Smith BP, Jafari D, Seamon MJ, Reilly $\mathrm{PM}$, et al. Resuscitative endovascular balloon occlusion of the aorta: assessing need in an urban trauma center. J Surg Res 2019;233:413-9.

2. Rabinovici R. Sixty-seven consecutive resuscitative thoracotomies by a single surgeon. Scand J Surg 2014;103:156-60.

3. Ladd AP, Gomez GA, Jacobson LE, Broadie TA, Scherer LR 3rd, Solotkin KC. Emergency room thoracotomy: updated guidelines for a level I trauma center. Am Surg 2002;68:421-4.

4. Brenner M, Bulger EM, Perina DG, Henry S, Kang CS, Rotondo 
MF, et al. Joint statement from the American College of Surgeons Committee on Trauma (ACS COT) and the American College of Emergency Physicians (ACEP) regarding the clinical use of resuscitative endovascular balloon occlusion of the aorta (REBOA). Trauma Surg Acute Care Open 2018;3:e00154.

5. Shin HK, Han HS, Lee TS, Park DJ, Jung KW, Kim KS. Resuscitative endovascular balloon occlusion of the aorta in a trauma patient with hypovolemic shock. Acute Crit Care 2015;30:115-8.

6. Park Y, Yu B, Lee G, Lee J, Choi K, Han A. Implementation of resuscitative endovascular balloon occlusion of the aorta at the Korean Regional Trauma Center. Hong Kong Journal of Emergency Medicine 2019 Aug 8. doi: 10.1177/1024907919866563 [Epub ahead of print].

7. Chang SW, Lee SW, Jo HC, Kim DH, Chang YR. A course on endovascular training for resuscitative endovascular balloon occlusion of the aorta (ET-REBOA): a pilot study for residents and specialists. Research Square 2019 Oct 17. doi: 10.21203/ rs.2.16178/v1 [preprint].

8. Stannard A, Eliason JL, Rasmussen TE. Resuscitative endovascular balloon occlusion of the aorta (REBOA) as an adjunct for hemorrhagic shock. J Trauma 2011;71:1869-72.

9. Kim DH, Chang SW, Matsumoto J. The utilization of resuscitative endovascular balloon occlusion of the aorta: preparation, technique, and the implementation of a novel approach to stabilizing hemorrhage. J Thorac Dis 2018;10:5550-9.

10. Biffl WL, Fox CJ, Moore EE. The role of REBOA in the control of exsanguinating torso hemorrhage. J Trauma Acute Care Surg 2015;78:1054-8.

11. Matsumura Y, Matsumoto J, Kondo H, Idoguchi K, Ishida T, Okada Y, et al. Early arterial access for resuscitative endovascular balloon occlusion of the aorta is related to survival outcome in trauma. J Trauma Acute Care Surg 2018;85:507-11.

12. Brenner M, Moore L, Teeter W, Hu P, Yang S, Wasicek P, et al. Exclusive clinical experience with a lower profile device for resuscitative endovascular balloon occlusion of the aorta (REBOA). Am J Surg 2019;217:1126-9.

13. Lamhaut L, Qasim Z, Hutin A, Dagron C, Orsini JP, Haegel A, et al. First description of successful use of zone 1 resuscitative endovascular balloon occlusion of the aorta in the prehospital setting. Resuscitation 2018;133:e1-2.

14. Morrison JJ, Rasmussen TE. Noncompressible torso hemorrhage: a review with contemporary definitions and management strategies. Surg Clin North Am 2012;92:843-58.

15. Cannon J, Morrison J, Lauer C, Grabo D, Polk T, Blackbourne L, et al. Resuscitative endovascular balloon occlusion of the aorta (REBOA) for hemorrhagic shock. Mil Med 2018;183(suppl 2):55-9.

16. Morrison J, Reva V, Lönn L, Matsumoto J, Matsumara Y, Holcomb J, et al. Resuscitative endovascular balloon occlusion of the aorta (REBOA). Top stent- the art of endovascular hybrid trauma and bleeding management. Sweden: Örebro University Hospital; 2017.

17. Beyer CA, Johnson MA, Galante JM, DuBose JJ. Zones matter: hemodynamic effects of zone 1 vs zone 3 resuscitative endovascular balloon occlusion of the aorta placement in trauma patients. Injury 2019;50:855-8.

18. Abe T, Uchida M, Nagata I, Saitoh D, Tamiya N. Resuscitative endovascular balloon occlusion of the aorta versus aortic cross clamping among patients with critical trauma: a nationwide cohort study in Japan. Crit Care Med 2016;20:400.

19. Moore LJ, Brenner M, Kozar RA, Pasley J, Wade CE, Baraniuk MS, et al. Implementation of resuscitative endovascular balloon occlusion of the aorta as an alternative to resuscitative thoracotomy for noncompressible truncal hemorrhage. J Trauma Acute Care Surg 2015;79:523-32.

20. Borger van der Burg BLS, van Dongen TTCF, Morrison JJ, Hedeman Joosten PPA, DuBose JJ, Hörer TM, et al. A systematic review and meta-analysis of the use of resuscitative endovascular balloon occlusion of the aorta in the management of major exsanguination. Eur J Trauma Emerg Surg 2018;44:535-50.

21. Brenner ML, Moore LJ, DuBose JJ, Tyson GH, McNutt MK, Albarado RP, et al. A clinical series of resuscitative endovascular balloon occlusion of the aorta for hemorrhage control and resuscitation. J Trauma Acute Care Surg 2013;75:506-11.

22. DuBose JJ, Scalea TM, Brenner M, Skiada D, Inaba K, Cannon J, et al. The AAST prospective aortic occlusion for resuscitation in trauma and acute care surgery (AORTA) registry: data on contemporary utilization and outcomes of aortic occlusion and resuscitative balloon occlusion of the aorta (REBOA). J Trauma Acute Care Surg 2016;81:409-19.

23. Teeter WA, Matsumoto J, Idoguchi K, Kon Y, Orita T, Funabiki $\mathrm{T}$, et al. Smaller introducer sheaths for REBOA may be associated with fewer complications. J Trauma Acute Care Surg 2016;81:1039-45.

24. Eliason JL, Myers DD, Ghosh A, Morrison JJ, Mathues AR, Durham L, et al. Resuscitative endovascular balloon occlusion of the aorta (REBOA): zone I balloon occlusion time affects spinal cord injury in the nonhuman primate model. Ann Surg 
2019 Jun 7. doi: 10.1097/SLA.0000000000003408 [Epub ahead of print].

25. Reva VA, Matsumura Y, Hörer T, Sveklov DA, Denisov AV, Telickiy SY, et al. Resuscitative endovascular balloon occlusion of the aorta: what is the optimum occlusion time in an ovine model of hemorrhagic shock?. Eur J Trauma Emerg Surg 2018;44:511-8.

26. Russo RM, Williams TK, Grayson JK, Lamb CM, Cannon JW, Clement NF, et al. Extending the golden hour: Partial resuscitative endovascular balloon occlusion of the aorta in a highly lethal swine liver injury model. J Trauma Acute Care Surg 2016;80:372-80; discussion 378-80.
27. Russo RM, Neff LP, Lamb CM, Cannon JW, Galante JM, Clement NF, et al. Partial resuscitative endovascular balloon occlusion of the aorta in swine model of hemorrhagic shock. J Am Coll Surg 2016;223:359-68.

28. Kuckelman JP, Barron M, Moe D, Derickson M, Phillips C, Kononchik J, et al. Extending the golden hour for zone 1 resuscitative endovascular balloon occlusion of the aorta: improved survival and reperfusion injury with intermittent versus continuous resuscitative endovascular balloon occlusion of the aorta of the aorta in a porcine severe truncal hemorrhage model. J Trauma Acute Care Surg 2018;85:318-26. 